\title{
SEROPREVALENCE OF HIV INFECTION IN PULMONARY TUBERCULOSIS PATIENTS.
}

\section{Dr. K.G.R.Mallan* \\ Dr.A.Fathahudeen}

Associate Professor, Department of Pulmonary Medicine, Government Medical college Ernakulam. *Corresponding Author

Professor and Head, Department of Pulmonary, critical care and sleep medicine, Government Medical college Ernakulam.

Dr. Manjula V.D.

Professor and Head, Department of Community Medicine, Government Medical college Ernakulam.

ABSTRACT Aim : To Determine the seroprevalence of Human immunodeficiency virus infection among Pulmonary Tuberculosis patients in a tertiary care teaching institution, in Ernakulam district of Kerala state in India. Methods : Study was conducted in Respiratory medicine department and integrated counselling and testing centre (ICTC) of Government Medical College, Ernakulam With approval of IEC after getting written informed consent, a cross sectional study was conducted among 384 pulmonary TB patients who were registered under the National TB elimination Programme (RNTCP). Result :The prevalence of HIV among study population was $1.3 \%$. There was no gender difference while the highest age group less than 30 followed by 30-60. The major respiratory pathology among study subjects was consolidation (42.7\%) followed by fibrosis(25.3\%).Consolidation was a predominant presenting pathology seen in female pulmonary TB patients.The sensitivity of sputum smear against CBNAAT was $60.4 \%$ and specificity was $83 \%$.

Conclusion : The prevalence is low compared with the national average of 3 to $3.4 \%$ HIV co infection among Tuberculosis patients during the study period.

KEYWORDS : Pulmonary TB, Seroprevalence, HIV, Respiratory Pathology

\section{BACKGROUND}

Tuberculosis and HIV are two closely related diseases.

Eight countries account for two thirds of the global TB population : India (27\%), China(9\%),Indonesia( $8 \%)$, the Philippines(6\%)Pakistan(6\%),Nigeria(4\%), Bangladesh (4\%), and south Africa (3\%). India (27\%), China (14\%) and the Russian Federation (9\%) account for major share of Drug resistance TB patients in the world.

As per Govt. of India National Tuberculosis Elimination Programme (NTEP) Annual report 2020 :

a. 24.04 lakh TB patients notified in $2019.14 \%$ rise over 2018 .

b. HIV testing of all notified TB patients increased to $81 \%$ (2019) from $67 \%(2018)$.

c. 79,144 deaths due to Tuberculosis were reported in 2019 .

d. The provision for HIV testing for all notified TB patients increased from $67 \%$ in 2018 to $81 \%$ compared to 2019.

e. The HIV co - infection rate among incident TB patients is estimated to be $3.4 \%$.Total 92,000 HIV associated TB patients have been estimated annually. By numbers India ranks $2^{\text {nd }}$ in the world and accounts for about $9 \%$ of the global burden of HIV - associated TB. The mortality in this group is very high and 9,700 people die every year among TB / HIV co-infected patients.

The prevalence of HIV in India is $0.22 \%$ among the adult population.PLHIV ( people living with HIV) are 21 (16 -27 )times at higher risk of developing TB. Nearly $25 \%$ of all deaths among PLHIV are estimated to be due to TB ${ }^{1}$. To mitigate the effect of dual burden of HIV and TB , NTEP and National AIDS control programme have developed a collaborative framework.

All TB patients are now routinely subjected to HIV testing,which is mandatory and implemented in all states provider initiated testing and counselling(PITC).HIV testing should be done early after TB diagnosis and results are informed to treatment provider so that there is early linkage to HIV care and support ${ }^{2}$. TB and HIV testing facilities to be located close to each other.
The life time risk of developing active TB is $10 \%$ for a HIV negative patients who is infected with tuberculosis.HIV increases the risk of progression from TB infection to TB disease and PLHIV's have a $60 \%$ lifetime risk of developing TB disease. The consequences of HIV for TB control include low cure rates, high case fatality rates, high default rates and increased emergence of drug resistance. Multi drug resistant Tuberculosis is associated with advanced HIV infection ${ }^{3}$.

Seroprevalence of HIV in Pulmonary TB patients in a particular demographic profile, is an indicator of the burden of HIV in that population and the spread of HIV can also be assesed. Information after data analysis , can lead to control measures, which will aid in strengthening HIV care and ART services to HIV positive TB patients.

OBJECTIVE: To estimate the seroprevalence of HIV infection in Pulmonary Tuberculosis patients, attending outpatients and inpatients of Respiratory Medicine of GMC Ernakulam

To describe the respiratory pathology in the above subjects

\section{MATERIALS AND METHODS}

Study area and design : Area is Government Medical college (GMC) Ernakulam and setting is in the Respiratory Medicine department, NTEP unit and Integrated counseling and testing centre(ICTC ) of GMC,Ernakulam.A cross sectional survey of patients comprising hospital population of GMC Ernakulam. Study was conducted for a two year period from $15^{\text {th }}$ September 2017.

Study population and Sampling technique : Out patients and In patients of GMC Ernakulam above the age of 14,registered under National TB Elimination programme ( old RNTCP ), with microbiologically confirmed or clinically diagnosed Pulmonary TB, both new and retreatment, were included. Seropositive patients referred from ICTC and registered as Pulmonary TB were excluded.

Sample size was determined using the formula for single population proportion based on the following assumptions : estimated $3.4 \%{ }^{1} \mathrm{HIV}$ Co infection among TB patients, $95 \%$ confidence interval, absolute error d as 2 , the calculated 
sample size was 328 ( $4 \mathrm{pq} / \mathrm{dxd}$ ). Taking $15 \%$ non response rate the final sample size was 377 , rounded to 380 .Finally 384 samples taken.

All consecutive patients diagnosed to have pulmonary tuberculosis were taken from the start of the study.

Study instrument :

Patients were interviewed with a structured questionnaire for the demographic profile and symptomatology with documentation of radiological presentation, sputum status and HIV status. HIV testing was done in ICTC Govt. $\mathrm{MCH}$,Ernakulam, with three test kits namely ComboAids, SD Bioline and AIDS scan, as per govt. of India , National AIDS control Organisation guidelines.

\section{Data Collection:}

Questionnaires were collected from the willing participants directly through interrogation and the relevant lab investigation results added. CT scan ,Ultrasonogram, Mantoux test and bronchoscopy, done whenever necessary .

Ethical Consideration : Ethical approval was obtained from the institutional Ethics committee. Written informed consent was obtained from each participant for the voluntary participation in the study.

\section{Statistical Analysis :}

Data was coded and entered in Microsoft Excel and analysed using SPSS software version 16.

\section{RESULTS:}

Table No 1:-Age group and sex Distribution of study subjects

\begin{tabular}{|c|c|c|c|}
\hline \multirow{2}{*}{ Age grp } & \multicolumn{2}{|c|}{ Sex } & \multirow{2}{*}{ Total } \\
\cline { 2 - 3 } & Male & Female & \\
\hline$<30$ & $24(60 \%)$ & $16(40 \%)$ & $40(100 \%)$ \\
\hline $30-60$ & $195(81.3 \%)$ & $45(18.7 \%)$ & $241(100 \%)$ \\
\hline$>60$ & $88(85.4 \%)$ & $76(19.8 \%)$ & $103(100 \%)$ \\
\hline Total & $308(80.2 \%)$ & $76(19.8 \%)$ & $384(100 \%)$ \\
\hline
\end{tabular}

Male predominance was noted among study subjects, with majority of patients in the 30 - 60 age group, followed by least in $>60$. (Table No.1).
Cough was the predominant symptom in $94.5 \%$ of patients followed by dyspnoea in $76.3 \%$ and weight loss in $74.4 \%$.

Table No.2:- Prevalence of HIV among study Population according to Age group

\begin{tabular}{l|c|c|c|}
\hline \multirow{2}{*}{ Age group } & \multicolumn{2}{|c|}{ HIV } & \multirow{2}{*}{ Total } \\
\cline { 2 - 3 } & Positive & Negative & \\
\hline$<30$ & $1(2.5 \%)$ & $39(97.5 \%)$ & $40(100 \%)$ \\
\hline $30-60$ & $4(1.7 \%)$ & $23(98.3 \%)$ & $241(100 \%)$ \\
\hline$>60$ & $0(0 \%)$ & $103(100 \%)$ & $103(100 \%)$ \\
\hline Total & $5(1.3 \%)$ & $379(98.7 \%)$ & $384(100 \%)$ \\
\hline$p=0.36$
\end{tabular}

Prevalence of HIV among study population was $1.3 \%$, with highest in the age group $<30(2.5 \%)$ followed by $30-60(1.7 \%)$ as depicted in Table No 2.

Table No.3:- Prevalence of HIV According to gender

\begin{tabular}{|c|c|c|c|}
\hline \multirow{2}{*}{ Gender } & \multicolumn{2}{|c|}{ HIV } & \multirow{2}{*}{ Total } \\
\cline { 2 - 3 } & Positive & Negative & \\
\hline Male & $4(1.3 \%)$ & $304(98.7 \%)$ & $308(100 \%)$ \\
\hline Female & $1(1.3 \%)$ & $75(98.7 \%)$ & $76(100 \%)$ \\
\hline Total & $5(1.3 \%)$ & $379(98.7 \%)$ & $384(100 \%)$ \\
\hline
\end{tabular}

There was no gender difference in prevalence of HIV among the study subjects.(Table No 3).

Table no.4:- Frequency of Respiratory Pathology among study subjects

\begin{tabular}{|l|l|l|}
\hline Respiratory pathology & Number & Percentage \\
\hline Fibrosis & 97 & 25.3 \\
\hline Consolidation & 164 & 42.7 \\
\hline Collapse & 10 & 2.6 \\
\hline Abcess & 10 & 2.6 \\
\hline Cavity & 53 & 13.8 \\
\hline Effusion & 31 & 8.1 \\
\hline Pneumothorax & 4 & 1 \\
\hline Hydropneumothorax & 15 & 3.9 \\
\hline Total & 384 & 100 \\
\hline
\end{tabular}

The predominant presenting Respiratory pathology was consolidation seen in $42.7 \%$ of patients followed by fibrosis in $25.3 \%$. (Table No. 4 ).

$\mathrm{P}=0.009$

Table No.5 :- Distribution of Respiratory pathology according to gender

\begin{tabular}{|c|c|c|c|c|c|c|c|c|c|}
\hline Gender & Fibrosis & Consolidation & Collapse & Abcess & Cavity & Effusion & $\begin{array}{c}\text { Pneumo } \\
\text { thorax }\end{array}$ & $\begin{array}{c}\text { Hydropneumo } \\
\text { thorax }\end{array}$ & Total \\
\hline Male & $88(28.6 \%)$ & $120(39 \%)$ & $9(2.9 \%)$ & $10(3.2 \%)$ & $40(13 \%)$ & $26(8.4 \%)$ & $2(0.6 \%)$ & $13(4.2 \%)$ & 308 \\
\hline Female & $9(11.8 \%)$ & $44(57.9 \%)$ & $1(1.3 \%)$ & $0(0 \%)$ & $13(17.1 \%)$ & $5(6.6 \%)$ & $2(2.6 \%)$ & $2(2.6 \%)$ & 76 \\
\hline Total & $97(25.3 \%)$ & $164(42.7 \%)$ & $10(2.6 \%)$ & $10(2.6 \%)$ & $53(13.8 \%)$ & $31(8.1 \%)$ & $4(1 \%)$ & $15(3.9 \%)$ & 384 \\
\hline
\end{tabular}

Consolidation, was a predominant presenting pathology seen in female pulmonary TB patients while fibrosis in males and it was statistically significant with p value 0.009. ( Table No.5).

Sputum smears of 298 study subjects were also tested with CBNAAT to find out the sensitivity and specificity of sputum Microscopy and found that sensitivity was $60.4 \%$ and specificity was $83 \%$ (Table No 6).

\section{DISCUSSION.}

Most of the previous studies , the prevalence of HIV in TB , has been estimated including extra pulmonary and pulmonary TB patients . Present study includes only pulmonary TB. This study was a provider initiated HIV testing and counselling.

Across sectional study ${ }^{4}$ carried out in 8 DOTS centres in Delhi, in 2012, estimated a HIV prevalence of $2.4 \%$ among TB patients with $1.6 \%$ HIV prevalence in Pulmonary TB patients including smear positive and smear negative cases as well as new and retreatment cases .2\% of TB patients were HIV infected, as per the Annual RNTCP report of 2012 .Our study , also a cross sectional survey, included only a single DOTS centre of a tertiary care teaching institution. We included MDR TB patients also, who were not included in this Delhi study where the time limit for defining the outcome variable was at the end of 5 months of treatment.

A study involving 4 different centres, was undertaken by Tuberculosis Research centre ( ICMR ) in Chennai, in 1992 1993 by Ramachandran et al, where 2 District TB centres, a TB sanatorium and a TB hospital were involved and wide variation in prevalence was noted in the different centres, with overall seroprevalence of $4.7 \%$ among TB patients ${ }^{5}$.

Retrospective Prevalence studies with Medical Records analysis have also been conducted, as done in a study ${ }^{6}$ confined to pulmonary TB patients alone, at the National Chest hospital in Jamaica, between 1995 -2001 , where male preponderance in the patients was noted with a HIV prevalence of $11.6 \%$.Screening tests preceeded confirmation of HIV by western blot.In our study ,In the serial testing strategy, when results are positive for the first test kit ( "Combo 
Aids" ), which has high sensitivity and specificity for the antibodies, if the first test become positive, two additional test kits are used for further screening, as well as differentiate HIVl \& HIV2 infection. Indeterminate results ( second or third test is negative ) are followed up after a period of three months. A study ${ }^{7}$ done at Govt. medical College Jammu in 2004 , revealed a seroprevalence of $1.6 \%$ among pulmonary TB patients.

A provider initiated HIV testing study of pulmonary and extra pulmonary cases, by Alladi Mohan et al , in a tertiary care teaching hospital in Tirupati, South India, in 2013 , Co infection with HIV was seen in $4.6 \%$ of incident TB patients, close to $5 \%$ of national level in 2013-2014. $7.2 \%$ of Pulmonary TB patients were HIV positive ${ }^{8}$. As in our present study ,new and retreatment cases were studied and patients registered under RNTCP (NTEP) included . 3 rapid test kits used. The study was confined to incident TB cases.

Our study included microbiologically confirmed cases as well as those that were clinically diagnosed under NTEP. Clinically diagnosed TB patients were excluded in some studies and only microbiologically confirmed Pulmonary TB patients included as was done in a National prevalence survey setting in Zambia'. In this Zambian study there was a high HIV testing refusal rate. In our study, no patient refused HIV testing thus avoiding selection bias. In a hospital based study at Puducherry ${ }^{10}$, in 2014, seropositivity (2.6\%) among pulmonary TB patients was studied, using NACO guidelines, only in sputum smear positive pulmonary TB cases.

In a prospective study conducted in Jodhpur ${ }^{11}$ on newly diagnosed untreated pulmonary and extra pulmonary TB patients by Grover $\mathrm{S}$ et al, seroprevalence among pulmonary TB patients was $3.4 \%$, but here patients aged $15-45$ year only were included whereas in our study all patients above the age of 14 were included.

Sentinel seroprevalence surveys have also been done on this topic, as in a 2017 Nepal cross sectional study ${ }^{12}$ where 6 sentinel centres across the country were surveyed. Prevalence of HIV among pulmonary TB cases was $2.8 \%$. Here also prevalence was comparatively higher in male patients. Sentinel surveillance was carried out in Sassoon General hospital Pune ${ }^{13}$, for HIV infection in TB patients initially from $1995-1997$ and subsequently at yearly intervals till 2000. Statistically significant increase in seroprevalence was noted in successive years. Such sentinel surveys throw light on the trends of the epidemic and in planning strategies for addressing various problems.Our study is a prevalence study pertaining to a particular period and this is the main indicator to be measured by the surveillance methods.

The adult HIV prevalence at National level has had a steady decline from 2001-2017 ${ }^{14}$. As per the 25 September 2019 up dated Annual India TB report , the number of HIV infected people who go on to develop TB is increasing in India. The co infection rate was $3 \%$ in 2018 and 2019 as per the respective Annual India TB reports. As per the "Global Tuberculosis report $2019^{\prime \prime}$ also $3 \%$ HIV positivity was estimated among new and relapse TB patients in India in 2018.

The Prevalence of HIV in our study population with Pulmonary TB was $1.3 \%$. The estimated national HIV Co infection rate was $3.4 \%$ among TB patients, both pulmonary and extra pulmonary as per India TB report 2020 . Though there are studies in various states on this prevalence, National statistics for prevalence of HIV among pulmonary TB patients are not available.

Risk factors for HIV infection were not studied. HIV 1 antibodies were detected in all of our seropositive patients
Ours was a facility based data on Co infection and this may not reflect the true epidemiological picture of the general Population.

\section{Acknowledgement}

The author would like to thank Dr. Manjula V.D. \& Dept. Of Community Medicine for encouragement \& contribution in statistical analysis. Study funded by SBMR.

\section{REFERENCES :}

1. India TB report 2020. Annual report.National Tuberculosis Elimination Programme.

2. Integrated HIV TB module 2016, NACO -CTD MoHW Govt.of India and Vihaan program guidelines. Page 25 and 53.

3. Telzak EE et al ,1995,New England journal of Medicine, 333(14): 907-11,

4. Dr.Vishal C Soyam et al. Jan - Feb 2016, Prevalence and socio-demographic correlates of HIV among tuberculosis patients of DOTS centre Delhi, Asian journal of Medical sciences ,vol 7, issuel, pg 53-58.

5. R Ramachandran et al.October 2003, Seroprevalence of HIV infection among Tuberculosis patients in Tamilnadu . . Indian J Medical Research 118,pp147151.

6. Alpaka PE et al.2006:19(1) Prevalence of HIV infection in patients with pulmonary Tuberculosis at the national chest hospital in Jamaica. PanamSaludPublica, pp 38-43.

7. R Bahl et al October 2007.Prevalence of HIV infection among patients of Pulmonary tuberculosis attending Chest Diseases Hospital jammu,Indian journal of Community Medicine , vol.32,No.4 page 288-289

8. Alladi Mohan et al. December 2017 ,Provider initiated HIV testing \&Counselling in incident tuberculosis cases under National TB programme conditions at a tertiary teaching hospital in Tirupati ,South India.Indian $\mathrm{j}$ of Med Res 146, pp774-779.

9. 2017 Mar 29,The Prevalence of HIV among Adults with Pulmonary TB at a population level in Zambia. BMC Infect Dis ; 17 (1):236.

10. RoshnaA, Gopal R, Saleem m .2015, Prevalence of Pulmonary Tuberculosis and HIV Co infection - A hospital based study at Puducherry . Indian J Microbiolol Res : 2(2): 126-127.

11. 2020,Human Immunodeficiency Virus infection amongst newly diagnosed tuberculosis patients and their clinico - radiological profile. A prospective study from Western India. JFMPC year, vol9,issue 5,pg.2475-2479.

12. jan - Mar; 17,Prevalence of Immunodeficiency virus infection among Tuberculosis patients in Nepal. Journal of Nepal health Research council 2019 (42) : $15-20$.

13. S Tripathi et al.2002,Indian journal of Tuberculosis ,49,17

14. National AIDS control Organisation Annual report 2018-2019 